\title{
Building Electronic Disease Surveillance Capacity in the Peruvian Navy with SAGES
}

\section{Shraddha Patel, Miles Stewart, Martina Siwek}

Johns Hopkins Applied Physics Laboratory, Laurel, Maryland, United States

\section{Objective}

To introduce SMS-based data collection into the Peruvian Navy's public health surveillance system for increased reporting rates and timeliness, particularly from remote areas, as well as improve capabilities for analysis of surveillance data by decision makers.

\section{Introduction}

In the past 15 years, public health surveillance has undergone a revolution driven by advances in information technology (IT) with vast improvements in the collection, analysis, visualization, and reporting of health data. Mobile technologies and open source software have played a key role in advancing surveillance techniques, particularly in resource-limited settings. Johns Hopkins University Applied Physics Laboratory (JHU/APL) is an internationally recognized leader in the area of electronic disease surveillance. In addition to the Electronic Surveillance System for the Early Notification of Community-based Epidemics (ESSENCE) used by several state and local jurisdictions and the CDC in the U.S., JHU/APL has also developed the Suite for Automated Global Electronic bioSurveillance (SAGES). SAGES is a collection of modular, open-source software tools designed to meet the challenges of electronic disease surveillance in resource-limited settings. JHU/APL is working with the Peruvian Navy health system to improve their electronic disease surveillance capabilities. The Peruvian Navy currently uses a SAGES-based system called Alerta DISAMAR that was implemented several years ago in an effort supported by the Armed Forces Health Surveillance Branch, and in collaboration with the Naval Medical Research Unit No. 6 (NAMRU-6). The system uses both webbased and IVR-based (interactive voice response) data collection from several Navy health facilities in Peru. For the present effort, JHU/APL is implementing a new SMS-based data collection capability for the Peruvian Navy.

\section{Methods}

JHU/APL is engaged with the Peruvian Navy Health System to upgrade the existing SAGES-based Alerta DISAMAR surveillance system which relies on remote data collection using IVR (interactive voice recording) technology, with a SAGES-based system that uses SMS (short message service) text messages for remote data collection. Based on Peruvian Navy requirements, JHU/APL created mobile data entry forms for Android smartphones using the SAGES mCollect application. SAGES mCollect is built using Open Data Kit open source tools along with added features such as 128-bit encryption and quality checks. The JHU/APL team engages closely with end users and other stakeholders to determine system requirements and to deploy the system, as well as to train end users and the system administrators who will need to maintain the system once it is deployed. The JHU/APL team, consisting of both information technology and public health expertise, conduct a country-level capabilities and needs assessment to address design considerations and operational end user requirements. This assessment takes into account the requirements and objectives of the Peruvian Navy, while keeping in mind infrastructure, cost, and personnel constraints. A pilot test of SMS - based data collection is currently underway with 10 health clinics within the Navy.

\section{Results}

Many challenges exist when implementing electronic disease surveillance tools in resource-limited settings, but using a tailored approach to implementation in which specific needs, constraints, and expectations are identified with stakeholders helps increase the overall adoption and sustainment of the system. JHU/APL believes SMS-based data collection will be more sustainable than IVR-based data collection for the Peruvian Navy.

\section{Conclusions}

JHU/APL is deploying a SAGES-based electronic disease surveillance system for the Peruvian Navy that has great potential to increase reporting rates from its health facilities as well as improve data quality and timeliness, thus resulting in greater awareness and enhanced public health decision making. 


\section{Acknowledgement}

This project is supported by the Uniformed Services University, Center for Global Health Engagement and is conducted in close coordination with United States Southern Command Surgeon's Office. 